\title{
Extraction of Digital Elevation Model using Stereo Matching with Slope-Adaptive Patch Transformation
}

\section{Jaehong Oh* and Changno Lee**}

Erratum to: KSCE Journal of Civil Engineering (2016) 20(7):2902-2909

DOI 10.1007/s12205-016-1735-3

The original version of this article unfortunately contained a mistake.

Errata:

1. The affiliation of the second author was written incorrectly.

Seoul National University

Will be corrected as :

Seoul National University of Science and Technology 